NOTA DE PESQUISA

\title{
AVALIAÇÃO DO POTENCIAL DE CONTROLE BIOLÓGICO DO Metarhizium anisopliae SOBRE Boophilus microplus EM TESTE DE ESTÁBULO
}

\author{
THIAGO C. BAHIENSE ${ }^{1}$; ÉVERTON K.K. FERNANDES ${ }^{2}$, ISABELE DA C. ANGELO ${ }^{1}$, WENDELL M. DE \\ S. PERINOTTO ${ }^{3}$; VÂNIA R.E.P. BITTENCOURT ${ }^{4}$
}

\begin{abstract}
BAHIENSE, T.C.; FERNANDES, E.K.K.; ANGELO, I.C.; PERINOTTO, W.M.S.; BITTENCOURT, V.R.E.P. [Evaluation of the biological control potential of Metarhizium anisopliae toward Boophilus microplus in pen trials.] Avaliação do potencial de controle biológico do Metarhizium anisopliae sobre Boophilus microplus em teste de estábulo. Revista Brasileira de Parasitologia Veterinária, v. 16, n. 4, p. 243-245, 2007. Departamento de Parasitologia Animal, Instituto de Veterinária, Universidade Federal Rural do Rio de Janeiro, Km 7 da BR 465, Seropédica, RJ 23890-000, Brasil. E-mail: vaniabit@ufrrj.br

The aim of the present study was to evaluate the capacity of the fungus Metarhizium anisopliae to control Boophilus microplus tick in pen trials. Infested calves were held in individual pen and treated with fungus suspension through aspersion bath. The results were evaluated based on ticks' mortality rate for 28 days after treatment, and on the analysis of biology of tick's samples which were transferred to an incubation chamber. It was reported 33\% of mortality during the total period analyzed, and the production of eggs and nutritional rates were decreased only for a short period after treatment.
\end{abstract}

KEY WORDS: Boophilus microplus, Metarhizium anisopliae, biological control.

\section{RESUMO}

Avaliou-se por meio de um teste de estábulo a capacidade de Metarhizium anisopliae em controlar o carrapato Boophilus microplus. Foram realizados tratamentos em bovinos estabulados, utilizando este fungo aplicando-se suspensão conidial em banhos por aspersão. Os resultados foram obtidos pelo percentual de mortalidade de carrapatos durante 28 dias após o tratamento e pelos índices biológicos após incubação destes em câmara climatizada. Constatou-se 33\% de mortalidade no período total observado, sendo o índice de produção de ovos e índice nutricional reduzidos somente em um curto período após o tratamento.

\footnotetext{
${ }^{1}$ Curso de Pós-graduação em Ciências Veterinárias (CPGCV), Universidade Federal Rural do Rio de Janeiro (UFRRJ), Km 7 da BR 465, Seropédica, RJ 23890-000. Bolsista CAPES.

${ }^{2}$ CPGCV, UFRRJ, Km 7 da BR 465, Seropédica, RJ 23890-000. Bolsista CNPq.

${ }^{3}$ Curso de Graduação em Medicina Veterinária, UFRRJ, Bolsista PIBIC (CNPq/UFRRJ).

${ }^{4}$ Departamento de Parasitologia Animal, Instituto de Veterinária da UFRRJ, Pesquisador CNPq. E-mail: vaniabit@ufrrj.br
}

PALAVRAS-CHAVE: Boophilus microplus, Metarhizium anisopliae, controle biológico.

O carrapato Boophilus microplus (Canestrini, 1887) (Acari: Ixodidae), sinonímia de Rhipicephalus (Boophilus) microplus (MURREL; BARKER, 2003) é um parasito obrigatório, encontrado principalmente parasitando bovinos. No Brasil, os prejuízos causados pelo parasitismo por B. microplus chegam a 2 bilhões de dólares por ano (GRISI et al., 2002). Enquanto o controle dos carrapatos for baseado principalmente no uso de produtos químicos, a pecuária enfrentará crises sucessivas por conseqüência da seleção de cepas resistentes, a menos que novas alternativas e métodos de controle sejam introduzidos. A solução mais lógica para o manejo da resistência é a redução no uso de acaricidas (WHARTON, 1967).

Os entomopatógenos, que são considerados como importantes fatores na redução da população de pragas, ocorrem naturalmente no ambiente, e podem ser introduzidos ou aplicados. Portanto há a necessidade da conservação da população desses agentes microbianos, e para tal é importante conhecer a 
compatibilidade entre os entomopatógenos e outras práticas utilizadas tanto na agricultura como na pecuária, para evitar perdas da eficácia do controle natural (ALVES et al., 2001). O fungo Metarhizium anisopliae (Metschnikoff, 1879) Sorokin, 1883 (Ascomycota: Nectriaceae) vem sendo considerado um agente promissor no controle microbiológico de carrapatos.

O objetivo deste trabalho foi avaliar a capacidade do fungo entomopatogênico M. anisopliae de controlar as diferentes fases parasitárias de uma cepa de carrapato em teste de estábulo, visando o aumento da produtividade e amenizando os danos ao meio ambiente.

O experimento foi desenvolvido no Departamento de Parasitologia Animal, Instituto de Veterinária da Universidade Federal Rural do Rio de Janeiro, localizada no município de Seropédica, RJ. Quatro bezerros, com idade entre 8 e 12 meses, foram infestados em dias alternados, com aproximadamente 2.000 larvas de Boophilus microplus. A infestação se iniciou 24 dias antes do tratamento, para que nesta data houvesse todos os estágios parasitários, sendo prosseguida nos dias +3 , +5 e +7 para avaliar o efeito residual do tratamento. A coleta e contagem das fêmeas ingurgitadas nos três dias antes do tratamento, foram para avaliar a capacidade individual do bezerro em relação ao desenvolvimento dos carrapatos, servindo então de parâmetro para a determinação do percentual de mortalidade, segundo Henderson e Tilton (1955). O isolado de M. anisopliae utilizado no experimento foi o ESALQ-959, isolado no Rio de Janeiro de cigarrinha da pastagem. A produção foi realizada fazendo-se a inoculação do fungo em sacos de polipropileno contendo arroz semicozido e autoclavados visando obtenção de grande quantidade de massa conidial (ALVES; PEREIRA, 1998). O experimento constou de dois grupos de tratamento, com duas repetições cada, que receberam banhos por aspersão: 1) com suspensão do fungo na concentração de $8,0 \times 10^{8}$ conídios/ml e 0,1\% de espalhante adesivo Tween 80 e 2) água e $0,1 \%$ de espalhante adesivo Tween 80. Utilizou se através de pulverizador costal da marca Jacto, modelo PJH. A viabilidade dos conídios foi de 97\% segundo metodologia de Alves e Pereira (1998).

Foram efetuadas contagens diárias dos carrapatos desprendidos do corpo dos animais a partir do dia -3 até o dia +28 após o tratamento, além da avaliação do índice de produção de ovos e índice nutricional dessas fêmeas, as quais foram incubadas em câmara climatizada $\left(27 \pm 1^{\circ} \mathrm{C}\right.$, URe" $80 \%$, fotofase de $12 \mathrm{~h}$ ). A temperatura média avaliada no período foi de $22^{\circ} \mathrm{C}$ e a umidade relativa foi de $70 \%$.

Apesar da variação do percentual de mortalidade, foi observado que houve um percentual médio de mortalidade de $33 \%$ no grupo tratado em relação ao grupo controle. A similaridade dos resultados até o dia +17 demonstrou haver o mesmo grau de suscetibilidade entre ninfas e adultos. As larvas foram as mais suscetíveis ao patógeno, pois a partir do dia +17 foram verificados elevados percentuais de mortalidade. Castro et al. (1997) em teste de estábulo com M. anisopliae e bovinos infestados com B. microplus observaram uma escala crescente de suscetibilidade envolvendo adultos, larvas e ninfas do carrapato. As elevadas mortalidades observadas a partir do dia +24 demonstraram haver atuação nas infestações subseqüentes ao tratamento, demonstrando assim um poder residual deste bioacaricida (Figura 1).

O índice nutricional foi reduzido no segundo dia após o tratamento e outra diferença em relação ao grupo controle foi observada no dia +25 após tratamento, entretanto com valor inferior ao grupo tratado. As demais coletas não demonstraram diferenças entre o grupo tratado e o controle em relação a esse parâmetro biológico. O índice de produção de ovos apresentou redução no segundo dia após tratamento. Em relação às demais coletas, apesar de haver algumas diferenças esse índice se apresentou menor no grupo tratado do que no grupo não tratado (Tabela 1). Experimentos anteriores in vitro já

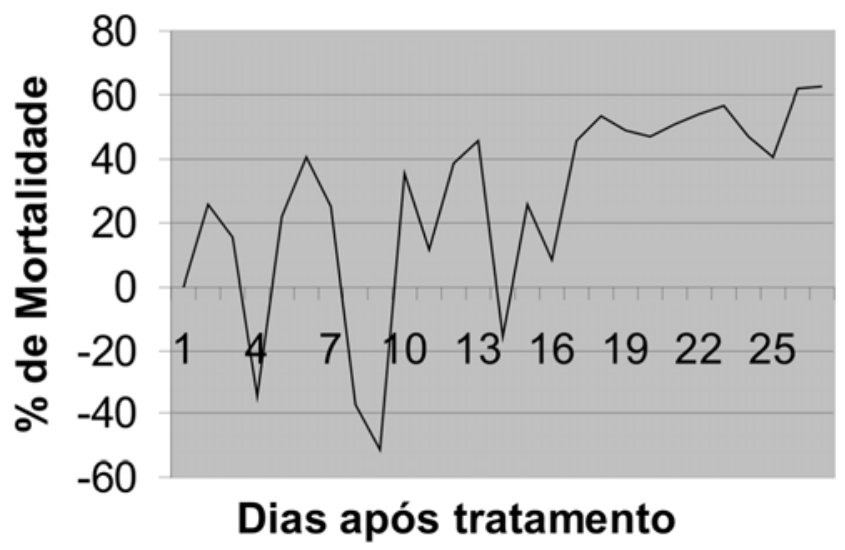

Figura 1. Mortalidade de Boophilus microplus, submetidos ao tratamento com Metarhizium anisopliae em teste de estábulo.

Tabela 1. Índice de Produção de Ovos (I.P.O.) e Índice Nutricional (I.N.), das fêmeas ingurgitadas provenientes de animais tratados com Metarhizium anisopliae em relação aos dias de coleta.

\begin{tabular}{cccccccccc}
\hline Parâmetros & Tratamentos & \multicolumn{7}{c}{ Dias de coleta após tratamento } \\
\cline { 3 - 9 } biológicos & & +2 & +4 & +6 & +9 & +11 & +13 & +17 & +25 \\
\hline I.P.O. & M.anisopliae & $39,4 \mathrm{a}$ & $51,7 \mathrm{a}$ & $51,3 \mathrm{~A}$ & $49,3 \mathrm{a}$ & $61,7 \mathrm{a}$ & $58,5 \mathrm{a}$ & $52,9 \mathrm{a}$ & $61,9 \mathrm{a}$ \\
& Controle & $58,6 \mathrm{~b}$ & $56,8 \mathrm{a}$ & $55,1 \mathrm{~A}$ & $55,4 \mathrm{a}$ & $58,9 \mathrm{~b}$ & $59,0 \mathrm{a}$ & $58,2 \mathrm{a}$ & $54,0 \mathrm{~b}$ \\
I.N. & M.anisopliae & $61,1 \mathrm{a}$ & $67,9 \mathrm{a}$ & $68,1 \mathrm{~A}$ & $67,4 \mathrm{a}$ & $84,1 \mathrm{a}$ & $79,5 \mathrm{a}$ & $71,2 \mathrm{a}$ & $81,6 \mathrm{a}$ \\
& Controle & $79,4 \mathrm{~b}$ & $79,0 \mathrm{a}$ & $78,2 \mathrm{~A}$ & $78,6 \mathrm{a}$ & $82,9 \mathrm{a}$ & $79,0 \mathrm{a}$ & $78,0 \mathrm{a}$ & $75,5 \mathrm{~b}$ \\
\hline
\end{tabular}

Médias seguidas da mesma letra, em uma mesma coluna, dentro de cada parâmetro, não diferem entre si, segundo o teste de Kruskal-Wallis, seguido do teste de te Student (pd"0,05). 
haviam demonstrado melhores resultados (BITTENCOURT et al., 1992), entretanto, essas diferenças podem ser explicadas devido à alta sensibilidade deste entomopatógeno aos fatores climáticos, tais como umidade relativa e temperatura, afetando não só a virulência como também epizootias. Quando se trata de métodos de controle biológico, menores percentuais de controle são esperados, quando comparados aos métodos convencionais com produtos químicos. Deve se ressaltar que essa diferença é compensada, pois o processo de controle biológico tem como objetivo manter a praga em níveis aceitáveis, além de preservar ao máximo o ambiente, com conseqüente preservação dos inimigos naturais dessa praga.

\section{REFERÊNCIAS BIBLIOGRAFICAS}

ALVES, S.B.; MOINO JR., A.; ALMEIDA, J.E.M. Produtos fitossanitários e entomopatógenos. In: ALVES, S.B. (ed.) Controle microbiano de insetos. Piracicaba: Fealq, 1998. p.217-238.

ALVES, S.B.; PEREIRA, R. M. Produção de fungos entomopatogênicos. In: ALVES, S.B. (ed.) Controle microbiano de insetos. 2 ed. Piracicaba: Fealq, 1998. p. 845-869.

BITTENCOURT, V. R. E. P. Ação do fungo Metarhizium anisopliae (Metschnikoff,1879) Sorokin,1883, sobre o carrapato Boophilus microplus (Canestrini,1887). 1992. $105 \mathrm{f}$. Tese (Doutorado) - Universidade Federal Rural do Rio de Janeiro, Rio de Janeiro, 2002.

CASTRO, A.B. A.; BITTENCOURT, V. R. E. P.; DAEMON, E.; VIEGAS, E. C. Eficácia in vivo do fungo Metarhizium anisopliae (isolado 959) sobre o carrapato Boophilus microplus em teste de estábulo. Revista Universidade Rural: Série Ciências da vida, v. 19, n. 1-2, p. 73-82. 1997.

GRISI, L.; MASSARD, C. L.; MOYA BORJA, G. E.; PEREIRA, J. B. Impacto econômico das principais ectoparasitoses em bovinos no Brasil. Hora Veterinária, v. 21, n. 125, p. 810, 2002.

HENDERSON, C. F.; TILTON, E. W. Tests with acaricides against the brow wheat mite. Journal of Economic Entomology, v. 48, n. 1, p. 157-161, 1955.

MURREL, A.; BARKER, S.C. Synonymy of Boophilus Curtice, 1891 with Rhipicephalus Koch, 1844 (Acari: Ixodidae). Systematic Parasitology, v. 56, n. 3, p. 169-172. 2003.

WHARTON, R.H. Acaricide resistance and cattle tick control. Australian Veterinary Journal, v. 43, n. 9, p. 394-398, 1967.

Recebido em 23 de janeiro de 2007.

Aceito para publicação em 09 de julho de 2007. 